\section{Risk Factors for Extended-Spectrum Beta-Lactamase-Producing Enterobacteriaceae Carriage Upon Pediatric Intensive Care Unit Admission}

To the Editor-The incidence of infections due to extendedspectrum $\beta$-lactamase (ESBL)-producing organisms is increasing in both children and adults. ${ }^{1}$ This increase is particularly concerning because ESBL-producing organisms are frequently resistant to antibiotics used in empiric sepsis regimens such as ceftriaxone, cefepime, and piperacillin-tazobactam. ${ }^{2}$ Additionally, plasmids carrying genes encoding ESBLs often harbor additional resistance mechanisms reducing the activity of aminoglycosides and fluoroquinolones. ${ }^{1,2}$ Thus, children with signs and symptoms of severe infections ultimately found to have infections with ESBL-producing organisms may not receive the most appropriate empiric therapy, increasing the likelihood of poor outcomes. ${ }^{3}$

Early recognition of ESBL colonization is important because colonization with ESBLs has been associated with subsequent invasive infections. ${ }^{4}$ Children warranting pediatric intensive care unit (PICU) admission are at particularly high risk for serious infections. An understanding of carriers of ESBLproducing organisms may play an important role in guiding appropriate empiric treatment.

We characterized risk factors for ESBL colonization among children admitted to the PICU by conducting a case-control study among patients admitted to The Johns Hopkins Hospital 45-bed tertiary-care PICU in Baltimore, Maryland. Rectal swabs were obtained from all children admitted to the unit between July 2014 and January 2015. Rectal swabs were inoculated into T-soy broth containing a 30- $\mu$ g ceftriaxone disk and incubated at $37^{\circ} \mathrm{C}$. Within 48 hours of inoculation, $100-\mu \mathrm{L}$ broth samples with visible turbidity were plated on MacConkey agar with a $30-\mu \mathrm{g}$ ceftriaxone disk and incubated at $37^{\circ} \mathrm{C}$ overnight. All recovered isolates within the zone of inhibition (inhibition zone, $<23 \mathrm{~mm}$ ) underwent routine identification and antimicrobial susceptibility testing using the BD Phoenix Automated System (Becton Dickinson Diagnostics, Sparks, MD).

Genomic DNA was extracted from isolates with ceftriaxone minimum inhibitory concentrations (MICs) of $\geq 2 \mu \mathrm{g} / \mathrm{mL}$ using the DNeasy Blood and Tissue Kit (Qiagen, Germantown, MD) and identification of $\beta$-lactamase-encoding genes was assessed using the Check-MDR CT103XL kit microarray-based assay (Check-Points, Wageningen, the Netherlands). Multilocus sequence typing (MLST) was used for amplification and sequencing of 8 housekeeping genes for E. coli and 7 housekeeping genes of K. pneumoniae (http://www.pasteur.fr/mlst).

Case patients were defined as children whose admission surveillance culture grew an ESBL-producing organism. Each case patient was matched to 3 control patients using a random number generator. Potential risk factors for colonization were collected on all patients. Data were extracted from all available inpatient and outpatient medical records from facilities within the Johns Hopkins Health System and from medical records of children who received care at institutions within the Epic Care Everywhere Network, a secure exchange that contains patient medical information from a large number of inpatient and outpatient healthcare networks throughout the United States. The Johns Hopkins University School of Medicine Institutional Review Board approved this study with a waiver of informed consent.

Baseline characteristics of cases and controls were compared using a $\chi^{2}$ or Fisher exact test for categorical variables and the Wilcoxon rank-sum test or Student $t$ test for continuous variables. $P$ values $\leq .05$ were considered significant. All analyses were performed using Stata, version 13 (StataCorp, College Station, TX).

In total, 854 rectal swabs from unique patients were obtained over the study period; 24 children were found to be colonized with ESBLs $(2.8 \%)$. bla $a_{\text {CTX-M genes were identified }}$ in all $21 \mathrm{E}$. coli isolates, in 1 of $2 \mathrm{~K}$. pneumoniae isolates, and in 1 of 1 E. cloacae isolates. A bla $a_{\mathrm{SHV}-12}$ gene was identified in the second K. pneumoniae isolate. Of the 24 ESBLs, 17 (71\%) contained $b a_{\text {CTX-M-15-like genes. The predominant circulating }}$ clonal strain was ESBL-producing E. coli ST131, which was identified in $63 \%$ of isolates.

The 24 ESBL-positive case patients were matched to 72 ESBL-negative control patients (Table 1). Within the previous 6 months, case patients were more likely to have had previous ESBL colonization or infection $(17 \%$ vs $1 \% ; P=.01)$ or to have been hospitalized in a high-ESBL-burden foreign country $(17 \%$ vs $1 \% ; P=.01)$, including China $(\mathrm{n}=1)$, India $(\mathrm{n}=1)$, Qatar $(n=1)$, and Saudi Arabia $(n=2)$. Case patients were more likely to have received recently chemotherapy (odds ratio [OR], 4.6; 95\% confidence interval [CI], 0.9-22.3) or a hematopoietic stem cell transplantation (OR, 10.1; 95\% CI, 1.0-102.7). Furthermore, 9 case patients (38\%) developed invasive infections with ESBL-producing organisms on a subsequent clinical culture (4 during the hospital admission and 5 within the subsequent 6 months). No control patients developed subsequent ESBL infections.

Our findings suggest that targeted screening of high-risk patients may be a reasonable consideration to identify ESBL colonization. Identifying children colonized with ESBLproducing organisms may be particularly relevant to help guide empiric antibiotic therapy because $40 \%$ of children colonized with ESBL-producing organisms at the time of PICU admission went on to develop invasive ESBL infections.

ESBL-producing bacteria have increasingly been identified in the community, which appears to be driven by clonal expansion of E. coli ST131 and person-to-person 
TABLE 1. Comparison of Children Colonized and Not Colonized With ESBL-Producing Enterobacteriaceae on Admission to a Pediatric Intensive Care Unit

\begin{tabular}{|c|c|c|c|c|}
\hline Characteristic & $\begin{array}{c}\text { ESBL Positive } \\
(\mathrm{n}=24), \text { No. }(\%)^{\mathrm{a}}\end{array}$ & $\begin{array}{c}\text { ESBL Negative } \\
(\mathrm{n}=72), \text { No. }(\%)^{\mathrm{a}}\end{array}$ & OR $(95 \% \mathrm{CI})$ & $P$ Value \\
\hline Age, median y (IQR) & $6.0(3.0-18.0)$ & $8.0(3.0-15.0)$ & $1.0(1.0-1.1)$ & .40 \\
\hline Male & $14(58.3)$ & $50(69.4)$ & $0.6(0.2-1.8)$ & .32 \\
\hline \multicolumn{5}{|l|}{ Race } \\
\hline White & $16(66.7)$ & $37(51.3)$ & $0.8(0.3-2.2)$ & .24 \\
\hline Black & $4(16.7)$ & $24(33.3)$ & $0.4(0.09-1.4)$ & .19 \\
\hline Asian & $2(8.3)$ & $2(2.8)$ & $3.2(0.2-45.6)$ & .26 \\
\hline Latino & $2(8.3)$ & $4(5.6)$ & $1.5(0.1-11.6)$ & .64 \\
\hline PRISM score, median (IQR) & $4.0(2.0-7.0)$ & $4.0(2.0-7.0)$ & $1.0(0.9-1.2)$ & .80 \\
\hline $\begin{array}{l}\text { Days from hospital admission to PICU } \\
\text { admission, median d (IQR) }\end{array}$ & $0.0(0.0-4.0)$ & $0.0(0.0-17.0)$ & $1.4(1.0-2.0)$ & .11 \\
\hline \multicolumn{5}{|l|}{ Reason for PICU admission } \\
\hline Planned surgical procedure & $11(45.8)$ & $32(44.4)$ & $1.1(0.4-2.9)$ & .90 \\
\hline Respiratory failure & $2(8.3)$ & $12(16.7)$ & $0.4(0.05-2.3)$ & .51 \\
\hline Trauma & $0(0.0)$ & $13(18.0)$ & $\ldots$ & .02 \\
\hline Sepsis & $5(20.8)$ & $2(2.8)$ & $9.2(1.3-100.8)$ & .01 \\
\hline Metabolic derangements & $1(4.2)$ & $4(5.6)$ & $0.7(0.01-8.0)$ & 1.00 \\
\hline Other $^{\mathrm{b}}$ & $5(20.8)$ & $8(11.1)$ & $2.1(0.5-8.3)$ & .23 \\
\hline \multicolumn{5}{|l|}{ Pre-existing conditions } \\
\hline Previously healthy & $3(12.5)$ & $28(38.9)$ & $0.2(0.04-0.9)$ & .02 \\
\hline Congenital heart disease & $1(4.2)$ & $9(12.5)$ & $0.3(0.01-2.4)$ & .44 \\
\hline Chemotherapy within the previous $6 \mathrm{mo}^{\mathrm{c}}$ & $4(16.7)$ & $3(4.2)$ & $4.6(0.95-22.3)$ & .05 \\
\hline HSCT within the previous $12 \mathrm{mo}$ & $3(12.5)$ & $1(1.4)$ & $10.1(1.00-102.6)$ & .03 \\
\hline Chronic steroid use or immunotherapy ${ }^{\mathrm{d}}$ & $1(4.2)$ & $2(2.8)$ & $1.5(0.02-30.4)$ & 1.00 \\
\hline Other $\mathrm{e}^{\mathrm{e}}$ & $0(0.0)$ & $2(2.8)$ & $\ldots$ & .41 \\
\hline \multicolumn{5}{|c|}{ Healthcare exposures in the 6 mo prior to PICU admission } \\
\hline Hospital admission & $12(50.0)$ & $29(40.3)$ & $1.5(0.5-4.2)$ & .40 \\
\hline PICU admission & $2(8.3)$ & $2(2.8)$ & $3.2(0.2-45.6)$ & .26 \\
\hline Hospitalization in a foreign country & $4(16.7)$ & $1(1.4)$ & $14.2(1.3-708.6)$ & .01 \\
\hline ESBL colonization or infection & $4(16.7)$ & $1(1.4)$ & $14.2(1.3-708.6)$ & .01 \\
\hline No. of hospital days, mean $( \pm S D)^{f}$ & $11.8( \pm 19.5)$ & $11.3( \pm 20.9)$ & $1.0(1.0-1.0)$ & .78 \\
\hline No. of antibiotic days, mean $( \pm S D)^{f}$ & $4.5( \pm 7.2)$ & $11.0( \pm 34.7)$ & $1.0(1.0-1.0)$ & .47 \\
\hline \multicolumn{5}{|c|}{ Antibiotic use in the 6 mo prior to PICU admission } \\
\hline Any antibiotic & $9(37.5)$ & $24(33.3)$ & $1.2(0.4-3.4)$ & .71 \\
\hline Penicillin & $6(25.0)$ & $12(16.7)$ & $1.7(0.4-5.6)$ & .36 \\
\hline Cephalosporins & $6(25.0)$ & $17(23.6)$ & $1.1(0.3-3.4)$ & .89 \\
\hline Carbapenems & $3(12.5)$ & $1(1.4)$ & $10.1(0.7-539.6)$ & .05 \\
\hline Fluoroquinolones & $2(8.3)$ & $1(1.4)$ & $6.4(0.3-385.9)$ & .15 \\
\hline Aminoglycosides & $3(12.5)$ & $5(6.9)$ & $1.9(0.3-10.7)$ & .39 \\
\hline
\end{tabular}

NOTE. ESBL, extended-spectrum $\beta$-lactamase-producing; OR, odds ratio; CI, confidence interval; IQR, interquartile range; PRISM, pediatric risk of mortality; PICU, pediatric intensive care unit; SD, standard deviation; HSCT, hematopoietic stemcell transplantation; SOT, solid organ transplant.

${ }^{\mathrm{a}}$ Unless otherwise noted.

${ }^{b}$ Other reasons for PICU admission included intractable seizures $(n=3)$, altered mental status $(n=2)$, burns $(n=2)$, myocarditis $(n=1)$, chemotherapy induction $(n=1)$, anemia $(n=1)$, cardiac arrest $(n=1)$, and allergic reactions $(n=2)$. ${ }^{c}$ Patients who received both chemotherapy and HSCT were only categorized as HSCT.

${ }^{\mathrm{d}}$ Excludes patients receiving immunotherapy for SOT, HSCT, or chemotherapy.

${ }^{\mathrm{e}}$ Other pre-existing conditions include end-stage renal disease on hemodialysis $(\mathrm{n}=1)$ and solid organ transplantation $(\mathrm{n}=1)$. ${ }^{\mathrm{f}}$ Calculation of the mean included only patients with the specific exposure.

transmission, sometimes in the absence of significant healthcare exposure. ${ }^{5-8}$ Our results indicate that previously healthy children are still at low risk for ESBL colonization. However, hospitalization in a foreign country is a strong predictor of ESBL colonization. This finding reflects that there are significant regional differences in ESBL prevalence, with a disproportionate burden in India, East and Southeast Asia, and the Middle East. ${ }^{9,10}$

This study has several limitations. This is a single-center study, and our findings must be repeated in a larger and more 
diverse setting. Additionally, although we completed a thorough review of inpatient and outpatient records from several healthcare facilities in Maryland, data may have been missing. However, this factor is expected to have been similar for both cases and controls.

As the incidence of ESBLs increases and they contribute to considerable morbidity and mortality, it is imperative to develop systems to identify children who are most at risk of infections caused by ESBL-producing organisms. Our findings suggest that in addition to reviewing prior culture histories to identify children with previous ESBL colonization or infection, targeted screening of children who received medical care abroad in high-risk countries, who recently received chemotherapy, or who recently underwent hematopoietic stem cell transplantations may be another strategy to help identify those most at risk for ESBL colonization.

\section{ACKNOWLEDGMENTS}

Financial support: The work was supported by funding the National Institutes of Health (grant no. K23-AI127935) awarded to P.D.T.

Potential conflicts of interest: All authors report no conflicts of interest relevant to this article.

\section{David X. Li, MD; ${ }^{1}$ \\ Anna C. Sick-Samuels, MD, MPH; Nuntra Suwantarat, MD, MPH; ${ }^{3}$ Rebecca G. Same, MD; ${ }^{2}$ Patricia J. Simner, $\mathrm{PhD} ;^{3}$ Pranita D. Tamma, MD, MHS $^{2}$}

Affiliations: 1. Division of Infectious Diseases, Department of Medicine, The Johns Hopkins University School of Medicine, Baltimore, Maryland; 2. Division of Pediatric Infectious Diseases, Department of Pediatrics, The Johns Hopkins University School of Medicine, Baltimore, Maryland; 3. Division of Medical Microbiology, Department of Pathology, The Johns Hopkins University School of Medicine, Baltimore, Maryland.

Address correspondence to Pranita D. Tamma, MD, MHS, The Johns Hopkins University School of Medicine, 200 North Wolfe St, Suite 3149, Baltimore, MD 21287 (ptamma1@jhmi.edu).

Infect Control Hosp Epidemiol 2018;39:116-118

(c) 2017 by The Society for Healthcare Epidemiology of America. All rights reserved. 0899-823X/2018/3901-0021. DOI: 10.1017/ice.2017.246

\section{REFERENCES}

1. Murray TS, Peaper DR. The contribution of extended-spectrum beta-lactamases to multidrug-resistant infections in children. Curr Opin Pediatr 2015;27:124-131.

2. Chandramohan L, Revell PA. Prevalence and molecular characterization of extended-spectrum-beta-lactamase-producing Enterobacteriaceae in a pediatric patient population. Antimicrob Agents Chemother 2012;56:4765-4770.

3. Sick AC, Tschudin-Sutter S, Turnbull AE, Weissman SJ, Tamma PD. Empiric combination therapy for gram-negative bacteremia. Pediatrics 2014;133:e1148-e1155.

4. Goodman K, Lessler J, Cosgrove SE, et al. A clinical decision tree to predict whether a bacteremic patient is infected with an ESBLproducing organism. Clin Infect Dis 2016;63:896-903.
5. Suwantarat N, Logan LK, Carroll KC, et al. The prevalence and molecular epidemiology of multidrug-resistant Enterobacteriaceae colonization in a pediatric intensive care unit. Infect Control Hosp Epidemiol 2016;9:1-9.

6. Madigan T, Johnson JR, Clabots C, et al. Extensive household outbreak of urinary tract infection and intestinal colonization due to extended-spectrum beta-lactamase-producing Escherichia coli sequence type 131. Clin Infect Dis 2015;61:e5-e12.

7. Logan LK, Braykov NP, Weinstein RA, Laxminarayan R, CDC Epicenters Prevention Program. Extended-spectrum $\beta$-lactamaseproducing and third-generation cephalosporin-resistant Enterobacteriaceae in children: trends in the United States, 1999-2011. J Pediatric Infect Dis Soc 2014;3:320-328.

8. Logan LK, Meltzer LA, McAuley JB, et al. Extended-spectrum $\beta$-lactamase-producing enterobacteriaceae infections in children: a two-center case-case-control study of risk factors and outcomes in Chicago, Illinois. J Pediatric Infect Dis Soc 2014;3:312-319.

9. Mathai D, Rhomberg PR, Biedenbach DJ, Jones RN, India Antimicrobial Resistance Study Group. Evaluation of the in vitro activity of six broad-spectrum beta-lactam antimicrobial agents tested against recent clinical isolates from India: a survey of ten medical center laboratories. Diagnost Microbiol Infect Dis 2002;44:367-377.

10. Tawfik AF, Alswailem AM, Shibl AM, Al-Agamy MH. Prevalence and genetic characteristics of TEM, SHV, and CTX-M in clinical Klebsiella pneumoniae isolates from Saudi Arabia. Microb Drug Resist 2011;17:383-388.

\section{Colistin-Resistant Klebsiella Infections Among Pediatric Oncology and Hematopoietic Stem Cell Transplantation Patients in Eastern India}

To the Editor-The epidemiology and clinical outcome of extremely drug-resistant (XDR) gram-negative bacterial (GNB) infections in the hemato-oncologic pediatric population is not well documented. Colistin-resistant Klebsiella (CRK) is one such XDR infection; it is resistant to most classes of antibiotics except 1 or 2 classes, and it is one of the most difficult pathogens to treat. These infections are increasingly common in settings with high XDR prevalence, and they are increasing globally because of travel, movement of food items, use in livestock, and widespread use of antibiotics (in patients and food animals). ${ }^{1-5}$ In the last 3 years, the trend in colistin resistance has increased among the commonly encountered GNB in our hospital (ie, for adults and pediatrics combined). From 2014 to 2016, the resistance to colistin in Klebsiella increased from 1.98\% to $3.12 \%$; in Escherichia coli, colistin resistance increased from $0.14 \%$ to $0.24 \%$; in Pseudomonas, colistin resistance increased from $0 \%$ to $0.87 \%$; and in Acinetobacter, colistin resistance increased from $0 \%$ to $4.49 \%$ (see the Figure online). In this retrospective study, we investigated the incidence, clinical presentation, and outcomes of CRK infection among pediatric oncology/transplantation patients (0-18 years old) in a cancer hospital in India between May 2011 and April 2017. 